\title{
Impact of average neutron energy on the fast neutron fluency measurement by Np237 fission to capture ratio and reverse dark current of planar silicon detector methods
}

\author{
M. Szuta ${ }^{1, *}$, S. Kilim ${ }^{1}$, E. Strugalska-Gola ${ }^{1}$, M. Bielewicz ${ }^{1,2}$, N.I. Zamyatin ${ }^{2}$, \\ A.I. Shafronowskaya ${ }^{2}, S$. Tyutyunnikov ${ }^{2}$, and $M$. Milewicz-Zalewska ${ }^{2}$ \\ ${ }^{1}$ National Centre for Nuclear Research, 05-400 Otwock-Świerk, Poland \\ ${ }^{2}$ Joint Institute for Nuclear Research, 141980 Dubna, Russia
}

\begin{abstract}
This work is a subsequent step to study the feasibility of fast neutron fluency measurements using two different complementary methods.

Np-237 samples and planar silicon detectors were mounted very close to each other on different sections of a subcritical assembly irradiated with the proton beam of $0,66 \mathrm{GeV}$ (the Quinta assembly at the Joint Institute for Nuclear Research, Dubna, Russia) to provide both samples with the same neutron fluency. We have processed the experimental data of irradiated Np-237 actinide samples and silicon detectors directly placed on two sections of the QUINTA setup without a lead shield-reflector.

Applying the try and error method we have found found that the neutron energy for which the ratio of the fission cross section to the capture cross section of the actinide Np-237 from the nuclear data base is equal to the measured ratio of the fissioned and captured actinide isotopes. The retrieved distinct fission and capture cross sections for the distinct neutron energy from the nuclear data base describe the average values.

The considered above experimental and earlier obtained data have been shown that the higher is the average neutron energy the smaller is the difference of the neutron fluency measurement between the two methods. This effect has been expected since the silicon detector method efficiently measures the fast neutrons of the energy higher than about $170 \mathrm{keV}$ while the actinide method covers a wider energy range.
\end{abstract}

\section{Introduction}

The feasibility study of the fast neutron fluency measurement using two different complementary methods was performed earlier [1]. Focusing on the efficiency dependence of incineration of the minor actinides at the distance of their samples from the neutron spallation source, we have measured, in fact, the impact of average neutron energy on the fast neutron fluency measurement by Np-237 fission to capture the ratio and reverse dark current by planar silicon detector methods.

So far, we could not place actinide samples in any other place but in the specimen window of the lead shield-reflector.

\footnotetext{
*e-mail: marcin.szuta@ncbj.gov.pl
} 
We have processed the experimental data of irradiated Np-237 actinide samples and silicon detectors directly placed on sections 2 and 4 of the target of QUINTA assembly (see Fig. 1) without the lead shield-reflector.
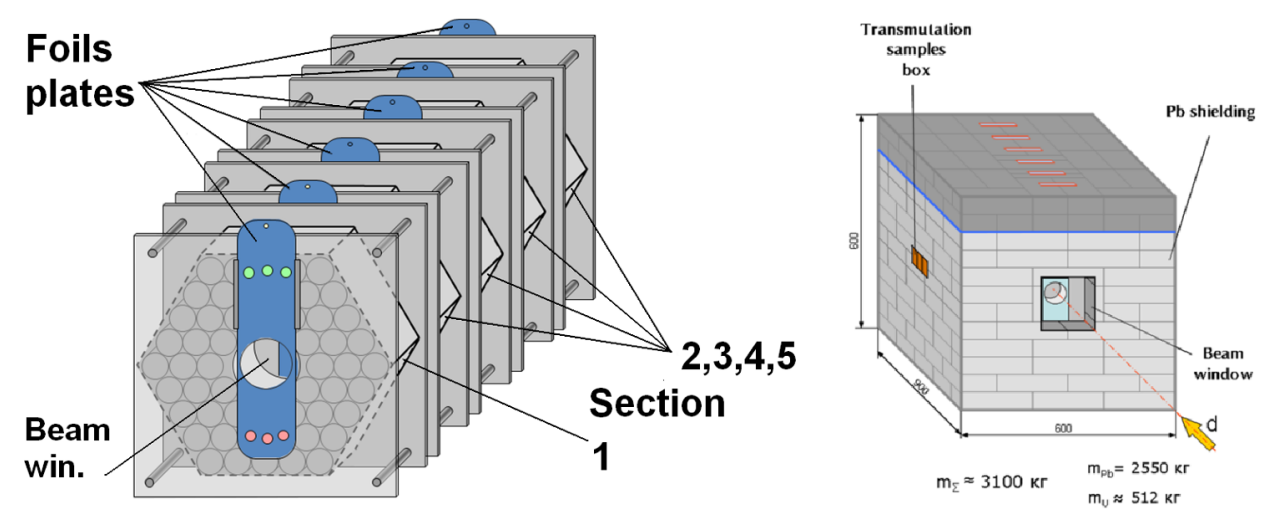

Figure 1. Scheme of Quinta assembly. On the left there is a view on the uranium target with supporting structures and plastics used for sample placement (detector's plates), on the right there is a view on the lead shielding enfolding the target with marked transmutation samples in the box (window) to locate actinides samples in the shielding

These samples were $12 \mathrm{~cm}$ from the neutron spallation source, which is about $8 \mathrm{~cm}$ closer than before when we placed the actinides samples in the window of the lead shield-reflector (see Fig. 1 on the right).

\section{Fast neutron fluency measuring methods}

The idea of the first method is to search for the neutron energy $\left(E_{d}\right)$ for the ratio $\left(\alpha\left(E_{d}\right)\right)$ of fission cross section $\left(\sigma_{f}\left(E_{d}\right)\right)$ to the capture cross section $\left(\sigma_{c}\left(E_{d}\right)\right)$ of the selected actinide isotope from the nuclear data base that is equal to the measured ratio $\left(\alpha_{m}\right)$ of the fissioned $\left(N_{y f}\right)$ and captured $\left(N_{y c} S\right)$ actinide isotopes (spectral indexes):

$$
\alpha\left(E_{d}\right)=\frac{\sigma_{f}\left(E_{d}\right)}{\sigma_{c}\left(E_{d}\right)}=\alpha_{m}=\frac{N_{y f}}{N_{y c}}=\frac{\overline{\sigma_{f}}}{\overline{\sigma_{c}}} .
$$

The idea of the second method is to measure fast neutron irradiation induced by the reverse dark current increase of the planar silicon detectors which is linearly proportional to the neutron fluency.

\subsection{Actinide fast neutron fluence measurement method}

Since the measured spectral indices are defined as the ratio of the average fission and capture cross sections, the retrieved distinct fission and capture cross sections for the distinct neutron energy from the nuclear data base describe the average values.

Having the average fission and capture cross section values we can evaluate the average neutron flux in the location of the actinide sample using the measured amount of the fissioned and captured actinide isotopes. Two different equations for fissioned $\left(N_{y f}\right)$ and captured $\left(N_{y c}\right)$ actinide isotopes should give the same average neutron flux value what is a proof for the correct measurement. 
The number of the neutron induced fissioned $\left(N_{y f}\right)$ and neutron captured actinide isotopes $\left(N_{y c}\right)$ in the actinide sample of volume $V_{p}$ can be expressed by the following equations:

$$
\begin{aligned}
& N_{y f}=V_{p} \bar{\Phi} N \overline{\sigma_{f}} t, \\
& N_{y c}=V_{p} \bar{\Phi} N \overline{\sigma_{c}} t,
\end{aligned}
$$

where: $\bar{\Phi}$ - average neutron flux in the place of actinide sample location $\left[\mathrm{n} / \mathrm{cm}^{2} \cdot \mathrm{s}\right], N-$ number of actinide isotopes in the volume unit $\left[\mathrm{cm}^{-3}\right], \overline{\sigma_{f}}, \overline{\sigma_{c}}$ - average microscopic cross section for the reactions (n, $\mathrm{f})$ or $(\mathrm{n}, \gamma)$ [barns], $t$ - irradiation time [s].

\subsection{Silicon semiconductor fast neutron fluence measurement method}

Fast neutrons with energy of $E_{n} \geq 100 \mathrm{keV}$ produce damage in the volume of the silicon semiconductor in the form of vacancies in the crystal lattice (knocking-out primary atom from the lattice site).

The silicon detector under neutron irradiation changes its electrical parameters (characteristics) due to generation of radiation defects in the crystal lattice. The radiation defects lead besides others to the reverse current growth.

The reverse dark current of the detector grows linearly $[2,3]$ with increasing the fast neutron fluency:

$$
\Delta I=\alpha_{I} \cdot V \cdot \Phi
$$

where: $\Delta I=\left(I_{\text {irrad }}=I_{\text {nonirrad }}\right)-$ increment detector current $[\mathrm{A}], \mathrm{V}-$ volume of the detector $\left[\mathrm{cm}^{-3}\right], \Phi-$ neutron fluency - equivalent for silicon damage fluency of fast neutrons with an energy of $1 \mathrm{MeV}\left[\mathrm{cm}^{-2}\right], \alpha_{I}=(5 \pm 10 \%) \cdot 10^{-17}-$ constant of radiation $\left[\mathrm{A} \cdot \mathrm{cm}^{-1}\right]$.

\subsection{The similarity of these two methods}

The mathematical forms of the equations describing the neutron fluency of the two methods are very much similar (see equations (1), (2) and (3)). In both methods the values which are proportional to the neutron fluency depend on the cross section of the neutron inducing damage in the silicon detector and cross sections of the neutron inducing fission and capture on Np-237 minor actinide (see Fig. 2). The cross sections used in both methods depending on the neutron energy are the primary reasons of the final result ( $\Delta I$ and $N_{y f}$ or $N_{y c}$ ) which enables us to evaluate the neutron fluency being searched for. Further similarity is that the volume of detectors of both methods is taken into account (consideration).

The neutron radiation induced damage of silicon detectors is also presented in Fig. 2. The values of the neutron damage cross section of silicon detectors in the function of neutron energy presented in Fig. 2, are extracted from the figure describing "Function of defect formation of $\mathrm{D}(\mathrm{E})$ in $\mathrm{MeV} \times \mathrm{mb}$, (Ougouag) and the function NIEL-FN-522 in $\mathrm{keV} \times \mathrm{cm}^{2} / \mathrm{g}$ ", presented in reference [4].

The damage cross section in the energy function starts with the threshold mode for the energy about $170 \mathrm{keV}$ [4].

\section{Measurement}

\subsection{Irradiation details}

The Quinta target was irradiated with a pulsed proton beam of $0.66 \mathrm{GeV}$ energy extracted from the Synchrotron accelerator, which is also located at JINR. The total number of protons of the irradiation is equal to $7.78 \cdot 10^{14}$ during the time of irradiation equal to 11890 seconds ( $5 \mathrm{~h} 15 \mathrm{~min}$ ). Prior to the irradiation, several polaroid films were placed on the front of Quinta to ensure the proton beam was striking in the centre of the beam window. 


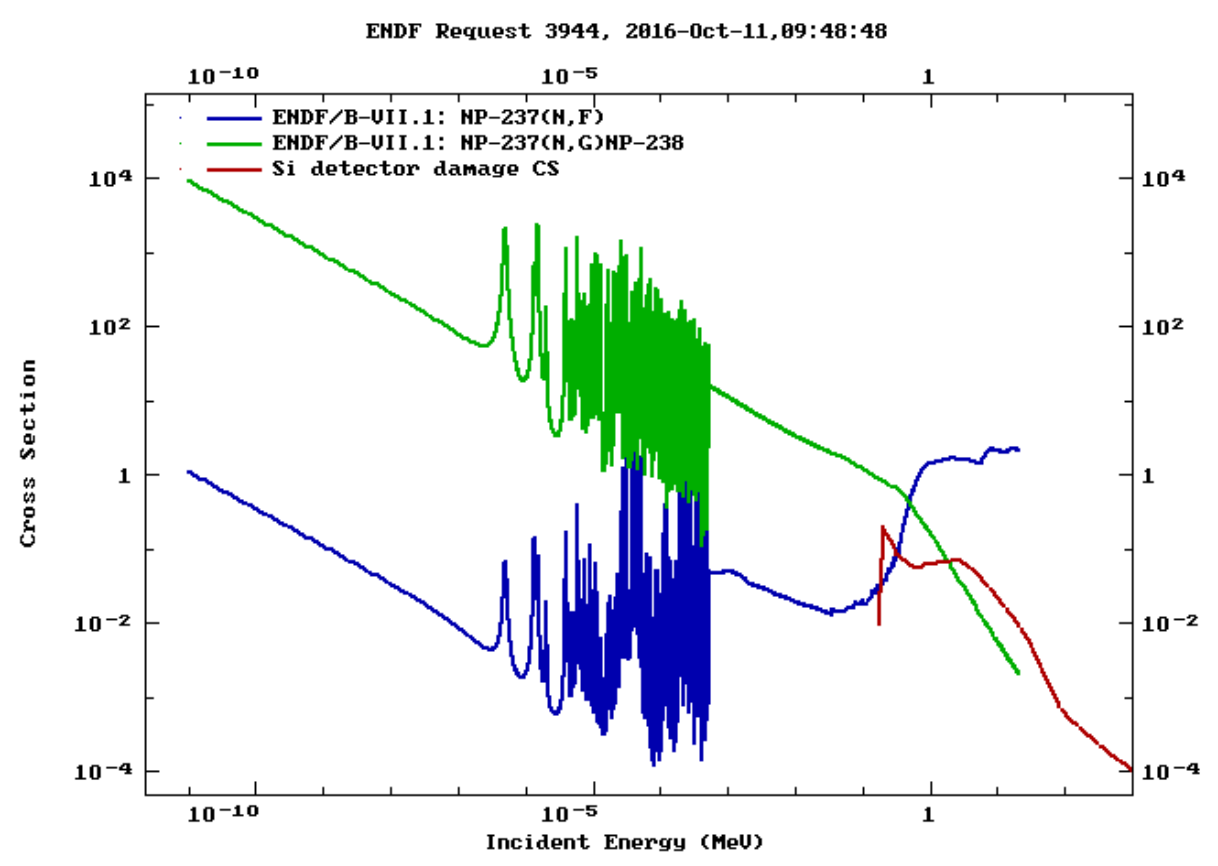

Figure 2. Cross section of neutron inducing damage in the silicon detector (red curve) and cross sections of neutron inducing fission and capture on $\mathrm{Np}-237$ minor actinide

\subsection{Results}

Gamma-ray spectrum analysis was carried out in a well established manner. The measured activities of the considered isotopes at the end of irradiation (EOI) were corrected for decay during the irradiation according to known deuteron beam profile, for the decay between EOI and start of counting (cooling time) and for the decay during the counting time. Dead time correction $\left(t_{\text {real }} / t_{\text {live }}\right)$ was also taken into account. Thus one can calculate the relative number of the considered produced isotopes at the end of irradiation for each measurement.

From gamma-ray spectrum of the irradiated Np-237 we have selected the following fission products ${ }^{92} \mathrm{Sr},{ }^{97} \mathrm{Zr},{ }^{132} \mathrm{I},{ }^{133} \mathrm{I}$ and ${ }^{135} \mathrm{I}$ which were used to evaluate the fission rate per one gram Np-237 and per one deuteron applying the proper equations.

Moreover, considering the actinide neptunium 237 samples after irradiation we have focused on the neutron capture $(\mathrm{n}, \gamma)$ reaction leading to production of $\mathrm{Np}-238$. From the gamma-ray spectrum of the irradiated $\mathrm{Np}-237$ we have selected five gamma ray lines, which were used to evaluate the capture rate per one gram of $\mathrm{Np}-237$ and per one deuteron.

In both cases of evaluation the fission and capture rates of $\mathrm{Np}-237$, the fission and capture rates of $\mathrm{Np}-238$ were disregarded as the small ones in comparison with the rates of $\mathrm{Np}-237$ during the transmutation analysis.

In Tab. 1 the collected experimental data of two irradiated Np-237 samples with proton beams of the energy $0.66 \mathrm{GeV}$ are presented.

Having the measured spectral index equal to the ratio of the average fission and average capture cross section we can evaluate the average neutron flux in the location of the actinide sample using the measured amount of the fissioned and captured actinide isotopes. 
Table 1. Compilation of incineration rate and capture rate of $\mathrm{Np}-237$ samples with proton beams of energy $0.66 \mathrm{GeV}$ results

\begin{tabular}{lll}
\hline Mass of NpO2 sample $[\mathrm{g}]$ & $\mathbf{1 . 1 1 9}$ & $\mathbf{1 . 1 4 7}$ \\
\hline Proton fluency & & $7.78 \times 10^{14}$ \\
\hline Mass of Np-237 in sample $[\mathrm{g}]$ & 0.987 & 1.011 \\
\hline Fission rate per gram sample and proton & $(2.82 \pm 0.418) \times 10^{-5}$ & $(1.26 \pm 0.319) \times 10^{-5}$ \\
\hline Capture rate per gram sample and proton & $(2.61 \pm 0.607) \times 10^{-5}$ & $(1.87 \pm 0.290) \times 10^{-5}$ \\
\hline Fission/Capture rate & $1.08 \pm 0.3$ & $0.67 \pm 0.20$ \\
\hline
\end{tabular}

Table 2. Comparison of neutron fluencies measured by two methods for two experiments

\begin{tabular}{llc}
\hline Sections of the QUINTA target & 2 & 4 \\
\hline $\begin{array}{l}\text { Neutron fluence estimated from the number of } \\
\text { fissions in the sample }\left[\mathrm{n} / \mathrm{cm}^{2}\right] \times 10^{13}\end{array}$ & 1.88 & 1.197 \\
\hline $\begin{array}{l}\text { Neutron fluence estimated from the number of } \\
\text { captures in the sample }\left[\mathrm{n} / \mathrm{cm}^{2}\right] \times 10^{13}\end{array}$ & 1.89 & 1.189 \\
\hline Average neutron energy $[\mathrm{MeV}]$ & 0.493 & 0.449 \\
\hline Neutron fluence measured by silicon detectors $\left[\mathrm{n} / \mathrm{cm}^{2}\right] \times 10^{13}$ & $\mathbf{1 . 5 9}$ & $\mathbf{0 . 6 8 6}$ \\
\hline
\end{tabular}

This has been done by applying the try and error method where we look for the neutron energy for which the ratio of the fission cross section to the capture cross section of the selected actinide isotope from the nuclear data base is equal to the measured ratio of the fissioned and captured actinide isotopes.

Since the measured ratio has been defined as the ratio of the average fission and capture cross sections, the retrieved distinct fission and capture cross sections for the distinct neutron energy from the nuclear data base (ENDF/B-VII.1) describe the average values.

Two different equations for the fissioned and captured actinide isotopes would give the same average neutron flux value (Eq. (1) and (2)) what is a proof for the correct measurement.

The obtained values for the average fission and capture cross sections have given an opportunity to evaluate the neutron fluencies by mean of the equations (Eq. (1) and (2)). This is collected in Tab. 2. Adding the values of the neutron fluencies measured by the silicon detector method we can compare the two methods. This is also shown in Tab. 2.

As it has been expected that the neutron fluencies evaluated by using the measured number of fissions in the sample and the measured number of captures in the sample give (nearly) the same values within the error accuracy (within the standard deviation) in both experiments what is a proof for correct measurements. The precision of this method is estimated to be around $25 \%$.

\section{Discussion}

The analysis of the experiment, presented in the paper [1] published two years earlier, when the average neutron energy was equal to $0.452 \mathrm{MeV}$ and the difference of the neutron fluency measurement between the two methods was about $35 \%$ is also valid for the current experiment. In the current experiment when the average neutron energy is equal to $0.449 \mathrm{MeV}$, the difference of the neutron fluency measurement between the two methods is about $42 \%$. While for the average neutron energy equal to $0.493 \mathrm{MeV}$, the difference of the neutron fluency measurement between the two methods is about $15 \%$. We can see a certain regularity in the measurements, the higher is the average neutron energy the smaller is the difference 
Table 3. Compilation of neutron fluencies measured by two methods for three experiments in terms of average neutron energy

\begin{tabular}{lrrr}
\hline Average neutron energy $[\mathrm{MeV}]$ & 0.493 & 0.452 & 0.449 \\
\hline $\begin{array}{l}\text { Neutron fluence estimated from the } \\
\text { number of fissions in the sample }\left[\mathrm{n} / \mathrm{cm}^{2}\right] \mathrm{x} 1013\end{array}$ & 1.88 & 1.67 & 1.197 \\
\hline $\begin{array}{l}\text { Neutron fluence estimated from the } \\
\text { number of captures in the sample }\left[\mathrm{n} / \mathrm{cm}^{2}\right] \times 1013\end{array}$ & 1.89 & 1.64 & 1.189 \\
\hline Neutron fluence measured by silicon detectors $\left[\mathrm{n} / \mathrm{cm}^{2}\right] \times 10^{13}$ & $\mathbf{1 . 5 9}$ & $\mathbf{1 . 1 1}$ & $\mathbf{0 . 6 8 6}$ \\
\hline $\begin{array}{l}\text { Difference of the neutron fluency } \\
\text { measurement between the two methods }[\%]\end{array}$ & 15 & 35 & 42 \\
\hline
\end{tabular}

of the neutron fluency measurement between the two methods [see Tab. 3]. This effect has been expected since the silicon detector method efficiently measures the fast neutrons of the energy higher than about $170 \mathrm{keV}$.

The conclusion that was made in the paper published earlier [1] that the difference of two fast neutron fluence measurements gives the estimation of neutron fluence for the neutron energy below $170 \mathrm{keV}$, as the additional approach, is still valid. The closer the minor actinides are placed to the spallation neutron source the more efficient is their incineration.

\section{Conclusion}

Minor actinide samples and planar silicon detectors can be used as neutron fluency detectors especially in the high neutron energy range which is difficult to measure.

Given the importance of high energy neutron measurement in the ADS (Accelerator Driven System) the actinide and silicon detectors could be a very useful tool to study the minor actinides incineration efficiency.

The difference of two fast neutron fluency measurements carried out by two methods presented above gives an additional estimation of neutron fluency for the neutron energy below $170 \mathrm{keV}$ - the additional approach.

The higher is the average neutron energy the smaller is the difference of the neutron fluency measurement between the two methods.

\section{References}

[1] M. Szuta, S. Kilim, E. Strugalska-Gola, M. Bielewicz, N.I. Zamyatin, A. I. Shafronovskaya, S. Tyutyunnikov, EPJ Web of Conferences 138, 10006 (2017). doi:10.1051/epjconf/201713810006

[2] N. I. Zamyatin, A. E. Cheremukhin, and A. I. Shafronovskaya, Phys. Part. Nucl. Lett. 14(5), 762-777 (2017)

[3] I.A. Golutvin et al., Preprint E14-95-97, Dubna (1995)

[4] A. Van Ginneken, Fermi Nat. Accelerator Lab. Report FN-522-1989 (1989) 